\title{
Reappraisal of the ligament of Henle (ligamentum inguinale internum mediale; Henle, 1871): a topohistological study using Korean foetuses
}

\author{
J.D. Yang ${ }^{1}$, H.P. Hwang ${ }^{1}$, J.H. Kim² ${ }^{2}$ G. Murakami ${ }^{3}$ J.F. Rodríguez-Vázquez4 , B.H. Cho ${ }^{1}$ \\ ${ }^{1}$ Department of Surgery and Research Institute of Clinical Medicine, Chonbuk National University Hospital, Jeonju, Korea \\ 2Department of Anatomy, Chonbuk National University Medical School, Jeonju, Korea \\ ${ }^{3}$ Division of Internal Medicine, Iwamizawa Kojin-kai Hospital, Iwamizawa, Japan \\ 4Second Department of Anatomy and Embryology, Faculty of Medicine, Complutense University, Madrid, Spain
}

[Received 14 November 2012; Accepted 20 January 2013]

Ligament of Henle is one of muscle-associated connective tissues of the rectus abdominis muscle, but it has been confused with the conjoint tendon (a common aponeurosis for insertion of the inferomedial end of the obliquus internus and transversus abdominis muscles). To reconsider the inguinal connective tissue structures, we examined 20 mid-term foetuses (10 males and 10 females) at approximately 14-20 weeks of gestation (crown rump length 100-170 mm). In female horizontal sections, we consistently found the ligament of Henle as a wing-like aponeurosis extending from the lateral margin of the rectus tendon behind the superficial inguinal ring. The ligament was separated from and located behind the conjoint tendon. In all male foetuses, instead of the ligament, the conjoint tendon was evident behind the superficial ring and it winded around the posterior aspect of the spermatic cord. Therefore, although a limited number of specimens were examined, the ligament of Henle was likely to be a female-specific structure. The ligament of Henle, if developed well, may provide an arch-like structure suitable for a name "falx inguinalis" instead of the inferomedial end of the conjoint tendon. In addition, a covering fascia of the iliopsoas muscle joined the posterior wall of the inguinal canal in male, but not in female, specimens. (Folia Morphol 2013; 72; 2: 147-154)

Key words: inguinal canal, rectus abdominis muscle, conjoint tendon, inguinal falx, gender difference, human foetus

\section{INTRODUCTION}

According to a famous textbook by Hollinshead [9], ligamentum inguinale internum mediale [8] or the ligament of Henle is a thickening in the lowermost part of the lateral border of the rectus sheath. The textbook also states about a confusion in Nomina Anatomica between the ligament of Henle and the conjoint tendon (conjoined tendon; Tendo conjunctivus; a common aponeurosis for insertion of the inferomedial end of the obliquus internus and transversus abdominis muscles): actually, there is no description on the ligament in Gray's anatomy and, instead, the conjoint tendon is considered to join the rectus sheath [17, 19]. A textbook by Bouchet and Cuilleret [5] clearly demonstrated the "ligament de Henle" as a wing-like extension of the anterior rectus sheath in the multiple line-drawings. In contrast, an excellent review by Skandalakis et al. [16] denied the existence of the ligament. Figure 1 displays a summary of

Address for correspondence: J.H. Kim, MS, PhD, Department of Anatomy, Chonbuk National University Medical School, San 2-20, Geumam-dong, Deokjin-gu, Jeonju 561-712, Republic of Korea, tel: +82-63-250-1570, fax: +82-63-250-6197, e-mail: 407kk@hanmail.net 


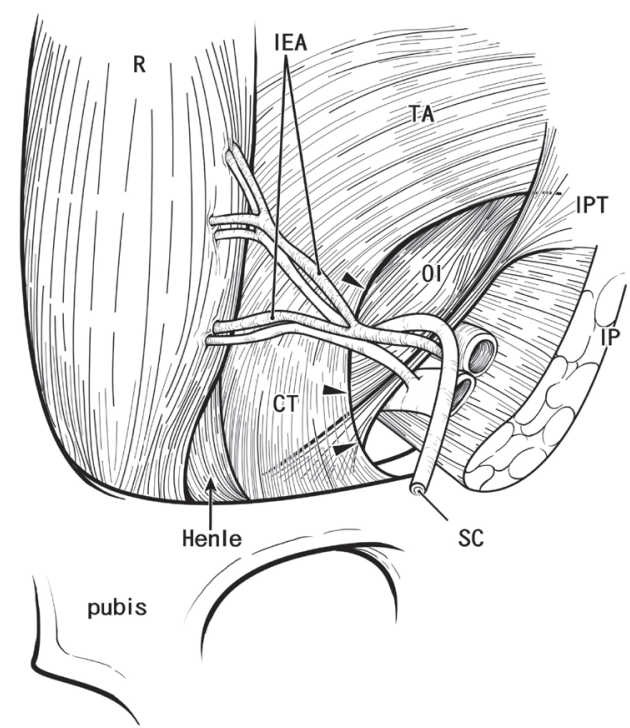

Figure 1. Schematic representation of the muscle-associated fibrous structures in the male inguinal region: a concept of the ligament of Henle. Viewed from the internal or peritoneal side of the abdomen. The transversalis fascia has been removed to show the ligament of Henle (Henle). The arch-like free margin (arrowheads) of the conjoint tendon (CT) corresponds to the falx inguinalis. The inguinal ligament is invisible behind the iliopubic tract (IPT); IEA inferior epigastric artery; IP — iliopsoas muscle; OI — obliquus internus muscle; $\mathrm{R}$ - rectus abdominis muscle (posterior aspect); $\mathrm{SC}$ - spermatic cord; TA — tranversus abdominis muscle.

the inguinal structures according to views that accepted the ligament of Henle.

A famous research group of the inguinal anatomy, including Drs. Anson and McVay, considered the ligament of Henle as a lateral expansion of the rectus tendon with a variable width: actually, at the inferior end, the rectus muscle sometimes (7 of 33 specimens) spreads laterally to attach to the pecten pubis by a broad tendon [2]. Thus, the ligament of Henle may provide an arch-like fibrous band to cover the posterior wall of the inguinal canal behind the superficial inguinal ring. However, at the medial-most site in the inguinal region, there is another, well known arch-like structure: the falx inguinalis. According to Gray's anatomy [17, 19], the falx inguinalis is considered as a synonym of the conjoint tendon. Likewise, Sen et al. [15] considered the falx as an arch-like part at the inferomedial edge of the conjoint tendon. We hypothesised that, if the foetal anatomy of connective tissues is simpler than adults, observations of the foetal inguinal region would provide a clue for reconsideration of those structures, the ligament of Henle, the conjoint tendon and the falx inguinalis. Consequently, using horizontal and sagittal sections of Korea mid-term foetuses, the aim of this study was to clarify topohistology of the muscle-associated structures in the medial part of the inguinal canal.

\section{MATERIALS AND METHODS}

In accordance with the provisions of the Declaration of Helsinki, 1995 (as revised in Edinburgh, 2000), we examined 20 mid-term foetuses (10 males and 10 females) at approximately $14-20$ weeks of gestation (crown rump length 100-170 mm). Five of the $10 \mathrm{ma}-$ le fetuses as well as 6 of the 10 female fetuses were processed for horizontal sections, whereas the other 9 for sagittal sections. These had been donated to the Department of Anatomy, Chonbuk National University in Korea with the agreement of the families concerned. Use of the foetuses for research had been approved by the University Ethics Committee. The foetuses had been obtained by induced abortions. After abortion, each of the mothers was personally informed by an obstetrician about the possibility of donating the foetus for research: no attempt was made to encourage donation. Because of randomisation of the specimen numbering, it was not possible to trace any of the families concerned. All specimens were normal, according to surface observations.

The specimens had been fixed in $10 \% \mathrm{v} / \mathrm{v}$ formalin solution. Depending on the size of the specimen, each abdominopelvic region was decalcified in $0.5 \mathrm{~mol} / \mathrm{L}$ EDTA, pH 7.5 (Decalcifiying solution B, Wako, Tokyo) for $1-3$ days at $4^{\circ} \mathrm{C}$. After routine procedures for paraffin embedded histology, 5 micron sagittal or horizontal sections, at 50-micron intervals, were prepared. Most sections were stained with hematoxylin and eosin, whereas 5-6 sections of every specimen underwent immunohistochemical staining for muscle fibers using mouse monoclonal anti-human desmin (dilution 1:50; Dako, Glostrup, Denmark) as the primary antibody. In human foetuses at the stages, desmin is strongly positive in striated muscle fibers, but it is also weakly positive in well differentiated smooth muscles [1]. The specimens were not autoclaved prior to treatment because of the loose nature of the fetal tissues. Prior to antibody staining, non-specific reactions were blocked by immersing the specimens in skim milk solution. Following incubation with primary antibody, the specimens were incubated with horseradish peroxidase (HRP)-labeled secondary antibody (Dako ChemMate EnVision Kit, Dako, Glostrup, Denmark), and antigen-antibody reactions were detected via the HRP-catalysed reaction with 


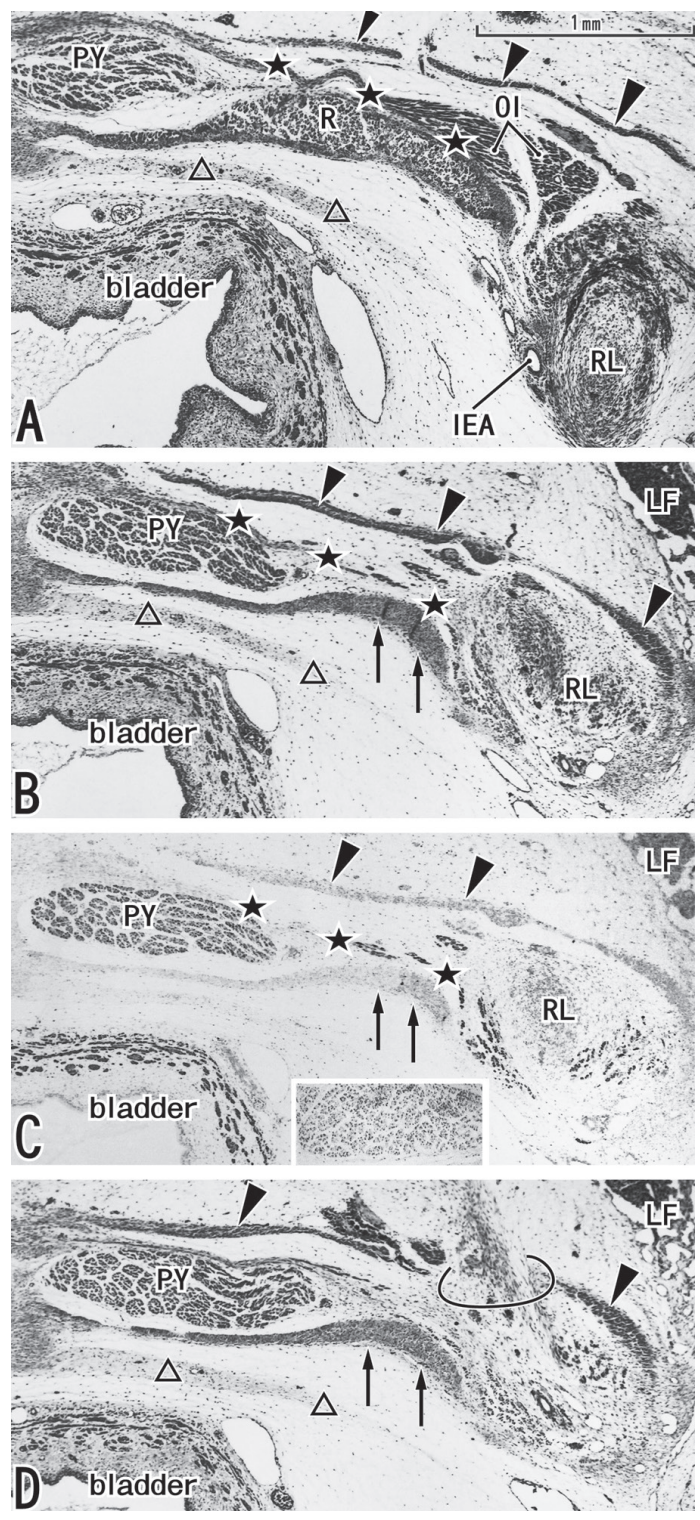

Figure 2. The rectus abdominis muscle provides an aponeurosis to reinforce the posterior wall of the inguinal canal in a female foetus. Horizontal sections of a female foetus at approximately 14 weeks of gestation (crown rump length, $105 \mathrm{~mm}$ ) stained with hematoxylin and eosin (panels A, B, D) and immunohistochemistry of desmin (panel $\mathbf{C}$ ). Panels $\mathbf{A}$ and $\mathbf{D}$ are the most superior and inferior sites, respectively, in the figure. Intervals between panels were $0.6 \mathrm{~mm}$ (A-B) and $0.2 \mathrm{~mm}$ (C-D). Panels B and C display near sections. Panels $\mathbf{A}$ and $\mathbf{D}$ contain the deep and superficial inguinal rings, respectively. The inferomedial end of the obliquus internus (OI) muscle attaches to a candidate conjoint tendon (stars in panels A-C): it extends to the anterior surface of the pyramidalis muscle (PY). The obliquus externus aponeurosis (arrowheads) makes a hole for the superficial ring (semicircle in panel D). Note that, at the inferolateral end, the rectus abdominis muscle $(R)$ provides a wing-like thick aponeurosis (arrows in panels B-D; ligament of Henle) to reinforce the posterior wall of the inguinal canal. This aponeurosis does not contain muscle fibers (panel C). A mesenchymal tissue band (triangles) is seen between in the bladder and rectus. Insert in panel $\mathbf{C}$ (pyramidalis muscle - PY) is a negative control without primary antibody. All panels were prepared at the same magnification (scale bar in panel A); IEA — inferior epigastric artery; LF — lymph follicle; RL — gubernaculum or the putative round ligament of the uterus. diaminobenzidine. The samples were subsequently counterstained with hematoxylin.

\section{RESULTS}

Throughout this description, the gubernaculum, a major component of the fetal inguinal canal, is called the spermatic cord or the round ligament of the uterus for easy understanding. To emphasize the presence of a well-developed fascia or aponeurosis originating from the inferolateral end of the rectus abdominis muscle, observations in females (Figs. 2, 3, 5) will be described prior to observations in males (Figs. 4,6) in each of the subsections below.

\section{Results in horizontal sections (Figs. 2-4)}

Horizontal sections were obtained from 11 foetuses ( 5 males, 6 females). In both females and males, the anterior rectus sheath was composed of 2 distinct fasciae. The external lamina was a thick aponeurosis for insertion of the obliquus externus muscle (we utilise the term "obliquus externus aponeurosis" throughout), whereas the internal lamina or the conjoint tendon arose from the inferomedial margin of the muscle bellies of the obliquus internus and transversus abdominis muscles. The contribution of the transversus muscle was limited, however, due to the high position of the inferior margin. The conjoint tendon extended medially along the anterior aspect of the pyramidalis muscle (Figs. 2A, 3C, 4B) and laterally along the posterior aspect of the round ligament of the uterus (Fig. 3C) or the spermatic cord (Fig. 4C). Immunohistochemistry displayed that the conjoint tendon contained striated muscle fibers (Figs. 2C, 3D) those were most likely to be derived from the obliquus internus muscle. The inferior part of the conjoint tendon was not clear in the smaller foetuses (Fig. 2B) in contrast to larger foetuses (Fig. 3E). The inferior part of the obliquus externus aponeurosis was divided into 3-4 pieces, whereas the inferolateral part was thick, forming the putative inguinal ligament. In addition, a mesenchymal tissue band, possibly corresponding to a primitive morphology of the transversalis fascia, extended along the left-right axis between the bladder and rectus of smaller foetuses at 14-16 weeks of gestation (Fig. 2), but such a band was replaced by a thin but definite fascia in larger foetuses (Fig. 3).

In sections of all 6 female foetuses, the inferolateral end of the rectus abdominis muscle consistently provided a thick fascia or aponeurosis extending laterally behind the superficial inguinal ring (Figs. 2B, D, 3G); morphologically, it was similar to a "wing" protruding 

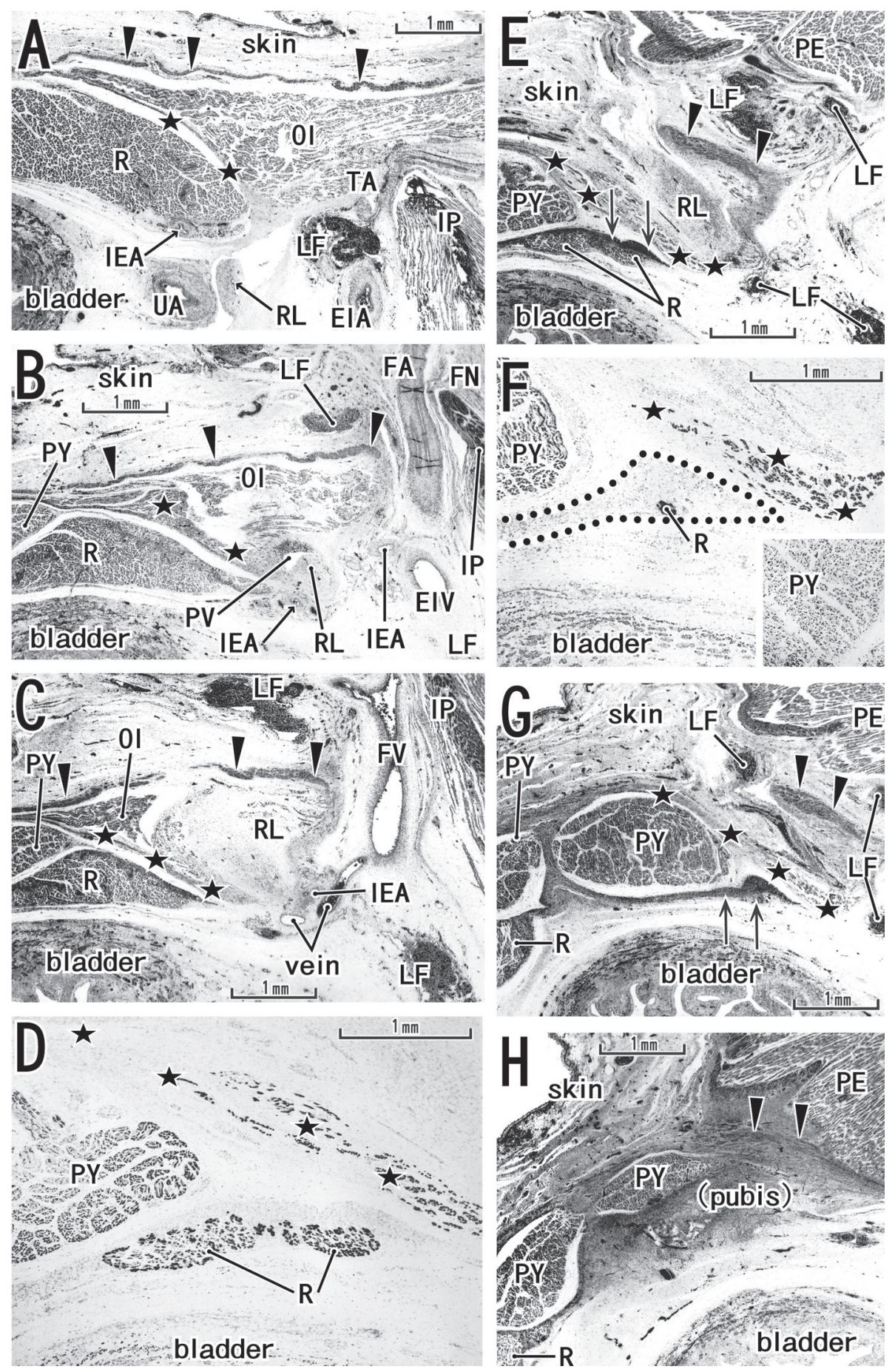

Figure 3. Topographical relation between the ligament of Henle and the conjoint tendon in a female foetus. Horizontal sections (slightly tilted) of a female fetus at approximately 18 weeks of gestation (crown rump length, $155 \mathrm{~mm}$ ) stained with hematoxylin and eosin (panels $\mathbf{A}-\mathbf{C}, \mathbf{E}$, $\mathbf{G}, \mathbf{H}$ ) and immunohistochemistry of desmin (panels $\mathbf{D}, \mathbf{F}$ ). Panels $\mathbf{A}$ and $\mathbf{H}$ are the most superior and inferior sites, respectively, in the figure. Intervals between panels were $0.9 \mathrm{~mm}(\mathbf{A}-\mathbf{B}), 0.4 \mathrm{~mm}(\mathbf{B}-\mathbf{C}, \mathbf{C}-\mathbf{E}), 0.3 \mathrm{~mm}(\mathbf{E}-\mathbf{G})$ and $0.5 \mathrm{~mm}(\mathbf{G}-\mathbf{H})$. Panels $\mathbf{D}$ and $\mathbf{E}(\mathbf{F}$ and $\mathbf{G})$ display near sections. Panel $\mathbf{A}$ includes the most inferior part of the transversus abdominis (TA) muscle. Panels $\mathbf{B}$ and $\mathbf{D}$ contain the deep and superficial inguinal rings, respectively. The inferomedial end of the obliquus internus (OI) muscle attaches to the conjoint tendon (stars) and, according to immunohistochemistry (panels D, F), the tendon contains abundant muscle fibers. The obliquus externus aponeurosis (arrowheads) is divided into 3-4 pieces, forming a thick inferior-most part (the putative inguinal ligament) in panels C-E. The ligament of Henle (arrows in panels $\mathbf{E}$ and $\mathbf{G}$ ) is evident at the inferolateral end of the rectus abdominis muscle (R) in the posterior or deep side of the conjoint tendon and, according to immunohistochemistry (panels $\mathbf{D}, \mathbf{F}$ ), contains muscle fibers of the rectus. Insert in panel $\mathbf{F}$ (pyramidalis muscle - PY) is a negative control without primary antibody; EIA and EIV — external iliac artery and vein; FA — femoral artery; FN — femoral nerve; FV — femoral vein; IEA — inferior epigastric artery; IP — iliopsoas muscle; LF — lymph follicle; PE — pectineus muscle; PV — process vaginalis of the peritoneal cavity; $\mathrm{RL}$ - gubernaculum or the putative round ligament of the uterus; UA — umbilical artery. 

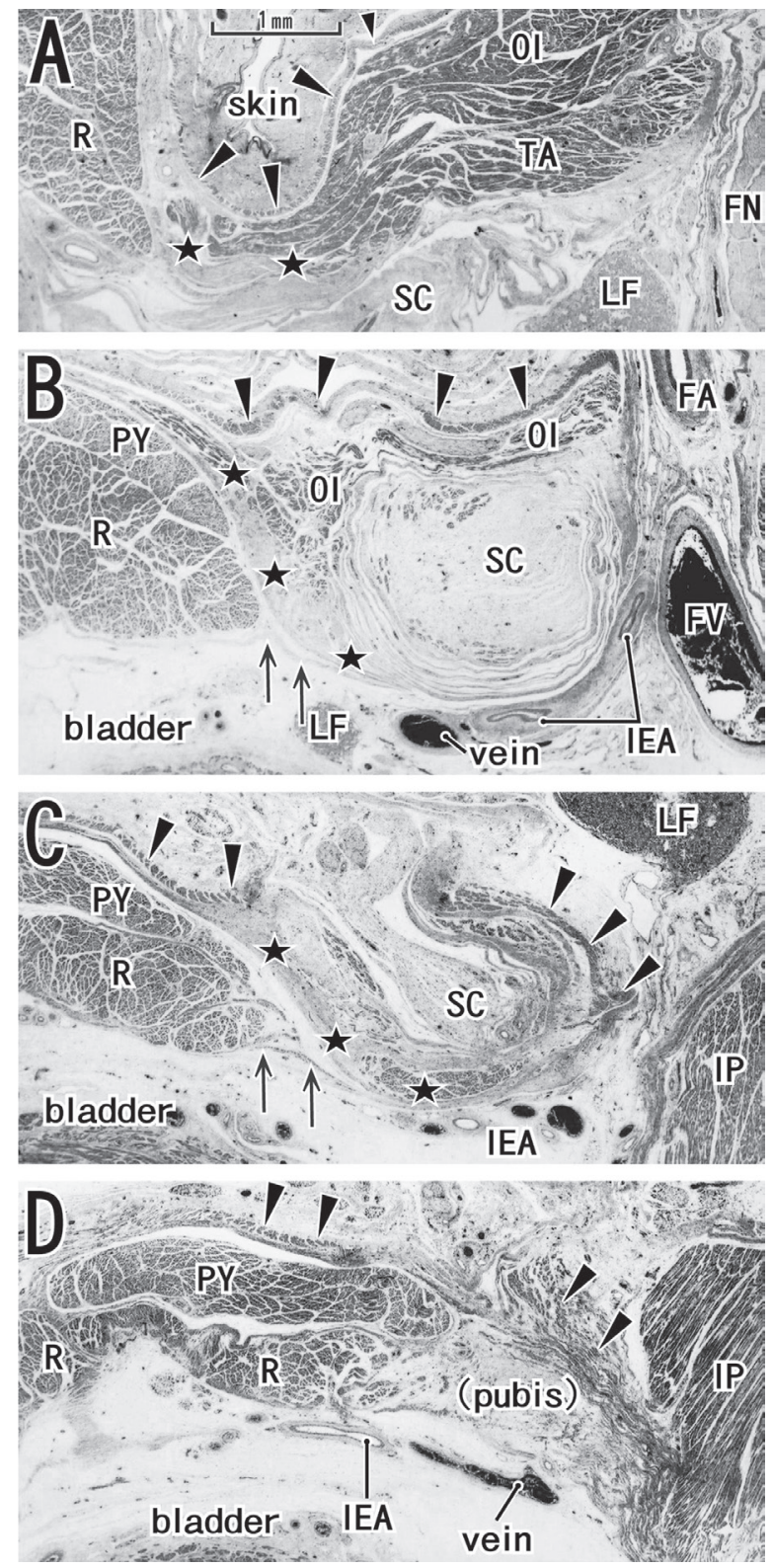

Figure 4. No wing-like aponeurosis is seen at the inferolateral end of the rectus abdominis muscle in a male foetus. Horizontal sections of a male foetus at approximately 20 weeks of gestation (crown rump length, $170 \mathrm{~mm}$ ) stained with hematoxylin and eosin. Panels $\mathbf{A}$ and $\mathbf{D}$ are the most superior and inferior sites in the figure, respectively. Intervals between panels were $1.6 \mathrm{~mm}(\mathbf{A}-\mathbf{B})$, $1.3 \mathrm{~mm}(\mathbf{B}-\mathbf{C})$ and $0.8 \mathrm{~mm}(\mathbf{C}-\mathbf{D})$. Panel $\mathbf{A}$ includes the most inferior part of the transversus abdominis muscle (TA). Panel $\mathbf{B}$ contains the inferior epigastric artery (IEA) crossing the gubernaculum or putative spermatic cord (SC) on the immediately medial side of the deep inguinal ring, while panel $\mathbf{C}$ includes the superficial inguinal ring. The conjoint tendon is indicated by stars in panels

A-C. The obliquus externus aponeurosis (arrowheads) is divided into 2 parts, forming a thick inferior-most part (the putative inguinal ligament) in panel $\mathbf{C}$ : it is located closely to (panel $\mathbf{C}$ ) and connected with (panel $\mathbf{D}$ ) a fascia covering the iliopsoas muscle (IP). Arrows in panels $\mathbf{B}$ and $\mathbf{C}$ indicate a thin fascia extending from the posterior aspect of the rectus abdominis muscle (R). All panels were prepared at the same magnification (scale bar in panel A). Other abbreviations are the same as in Figure 3. laterally from the rectus tendon and inserted into the pubis. Part of this wing-like aponeurosis was thick, with a tendon-like appearance along the supero-inferior axis: we identified it as the ligament of Henle. Immunohistochemistry demonstrated striated muscle fibers in the ligament of Henle in larger foetuses (Fig. 3F): they corresponded to the inferior end of the rectus abdominis muscle belly. The ligament was located on the inferior side of the origin of the inferior epigastric artery and $0.3-0.5 \mathrm{~mm}$ superior to the cartilaginous pubis. The conjoint tendon was separated from and located in the superficial side of the ligament (Figs. 2B, 3G). The obliquus externus aponeurosis covered the anterior or superficial aspect of the round ligament of the uterus (Figs. 2D, 3C, E) and was connected to the covering fascia of the pectineus muscle (Fig. $3 \mathrm{H}$ ). The iliopsoas muscle in females did not face the inguinal canal.

In contrast to the female foetuses, we did not find a wing-like aponeurosis from the rectus abdominis muscle in horizontal sections of the 5 male foetuses examined. Rather, we observed a thin fascia extending from the posterior aspect of the muscle toward the posterior aspect of the spermatic cord (Fig. 4B, C). The obliquus internus and transversus abdominis muscles were well developed and their thickness was maintained even at the inferomedial site near the spermatic cord. These muscles appeared to be continuous with a muscle band around the spermatic cord (Fig. 4B, C). The iliopsoas muscle was thick and approached the spermatic cord near the femoral artery and vein. Thus, the covering fascia of the iliopsoas muscle in males was connected to the inferomedial end of the obliquus externus aponeurosis or the putative inguinal ligament (Fig. 4D).

\section{Results in sagittal sections (Figs. 5, 6)}

Sagittal sections were made from 5 male and 4 female foetuses. In both genders, the rectus tendon was evident in the sagittal sections, being long and extending inferiorly on the posterior and lateral sides of the pyramidalis muscle. The conjoint tendon was consistently observed originating from the inferomedial margin of the obliquus internus muscle. The tendon extended along the anterior or superficial aspect of the rectus muscle toward the inferoposterior aspect of the round ligament of the uterus (Fig. 5D) or the spermatic cord (Fig. 6E, F). The conjoint tendon became thick and loose (triangles in Figs. 5, 6) and winded around the posterior aspect of the spermatic cord or round ligament. The obliquus externus aponeurosis had a thick, free, inferior end facing the femoral vessels 

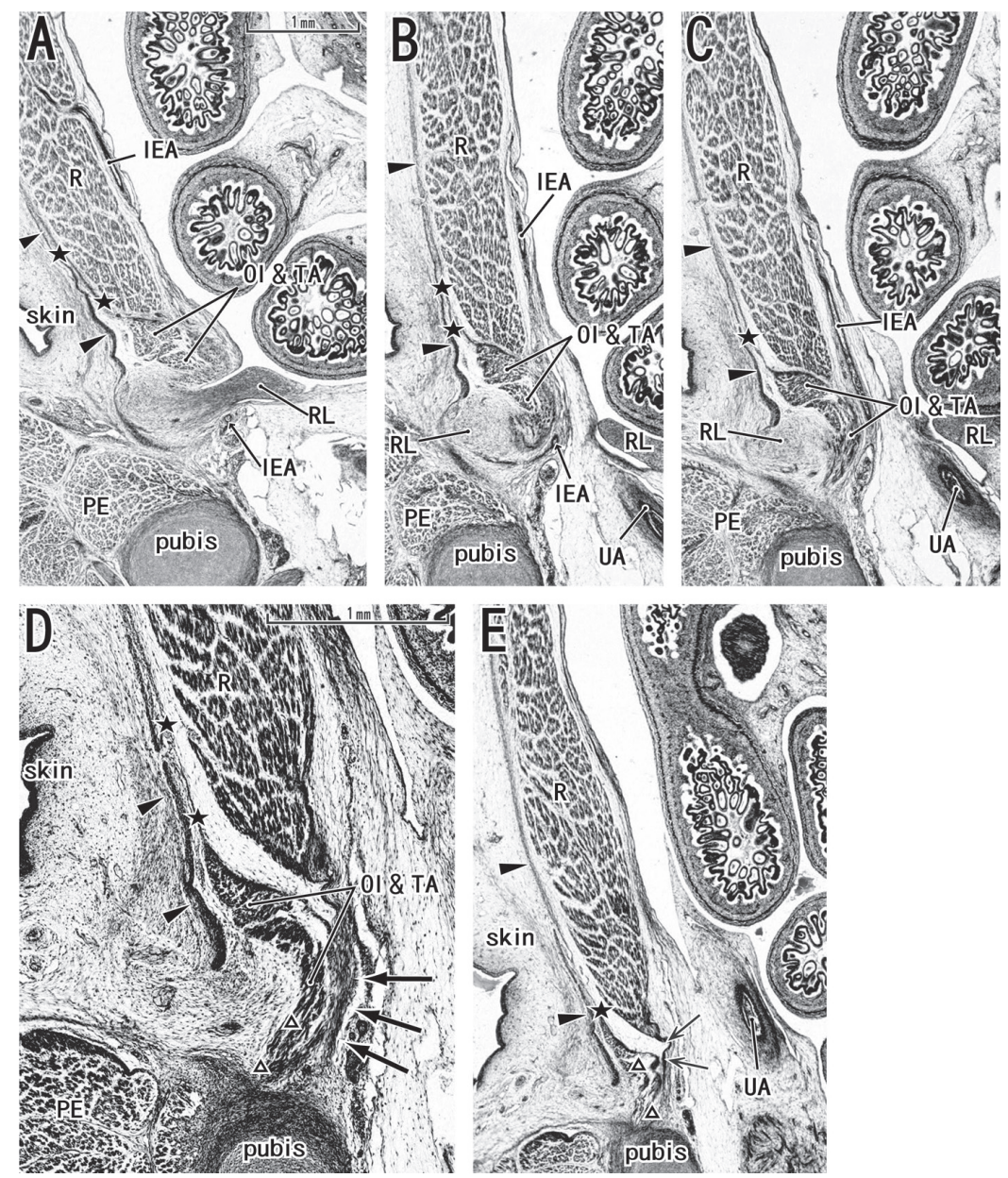

Figure 5. The rectus abdominis muscle reaches a lateral site behind the deep inguinal ring in a female foetus. Sagittal sections of a female foetus at approximately 15 weeks of gestation (crown rump length, $100 \mathrm{~mm}$ ) stained with hematoxylin and eosin. Panels $\mathbf{A}$ and $\mathbf{E}$ are the most lateral and medial sites in the figure, respectively. Intervals between panels were $0.3 \mathrm{~mm}(\mathbf{A}-\mathbf{B}), 0.2 \mathrm{~mm}$ (B-C, C-D) and $0.3 \mathrm{~mm}$ (D-E). Panels $\mathbf{A}$ and $\mathbf{D}$ contain the deep and superficial inguinal rings, respectively. The rectus muscle (R) reaches a lateral site above the deep inguinal ring (panel A). The obliquus internus (OI) and transversus abdominis (TA) muscles provide fragments at the inferomedial end, which extend along the posterior aspect of the putative round ligament (RL) of the uterus (panels $\mathbf{B}-\mathbf{D}$ ). The conjoint tendon (stars) extends inferiorly on the deep side of the external obliquus aponeurosis (arrowheads) to connect to a thick fascia (triangles in panels $\mathbf{D}$ and $\mathbf{E}$ ) behind the superficial ring. A thick fascia of the rectus muscle (long arrows in panel $\mathbf{D}$; a candidate ligament of Henle) is present on the lateral side of the inserted tendon (arrows in panel $\mathbf{E}$ ). Panels $\mathbf{A}-\mathbf{C}$ and $\mathbf{E}$ were prepared at the same magnification, while panel $\mathbf{D}$ was prepared at a slightly higher magnification (scale bars in panels $\mathbf{A}$ and $\mathbf{D}$ ). UA — umbilical artery. Other abbreviations are the same as in Figure 3.

(Fig. 6A) and was connected with the covering fascia of the pectineus muscle (Figs. 5A, 6C).

In the 4 female fetuses (Fig. 5), the rectus abdominis muscle extended more laterally than the deep inguinal ring: such a lateral expansion was difficult to identify in horizontal sections. In 4 of the 5 male foetuses, however, the rectus muscle was restricted to the medial side of the deep ring (Fig. 6). In the female specimen, a thick fascia continuous with the rectus tendon was evident behind the superficial inguinal ring (Fig. 5D); this fascia was short along the supero-inferior axis (less than $1 \mathrm{~mm}$ ) but appeared to correspond to the ligament of Henle seen in the horizontal sections. Actually, this fascia was separated from and located in the immediately deep side of the conjoint tendon. However, it was not evident in males.

\section{DISCUSSION}

The present histology demonstrated that the ligament of Henle was likely to be a constant morphology in female foetuses. In contrast, we found no evidence of the wing-like aponeurosis in male foetuses, although the number of specimens was limited. The ligament seemed to be a thickening of the rectus tendon rather than a part of the rectus sheath, because it contained muscle fibers. Actually, Condon [6] described the pubic 

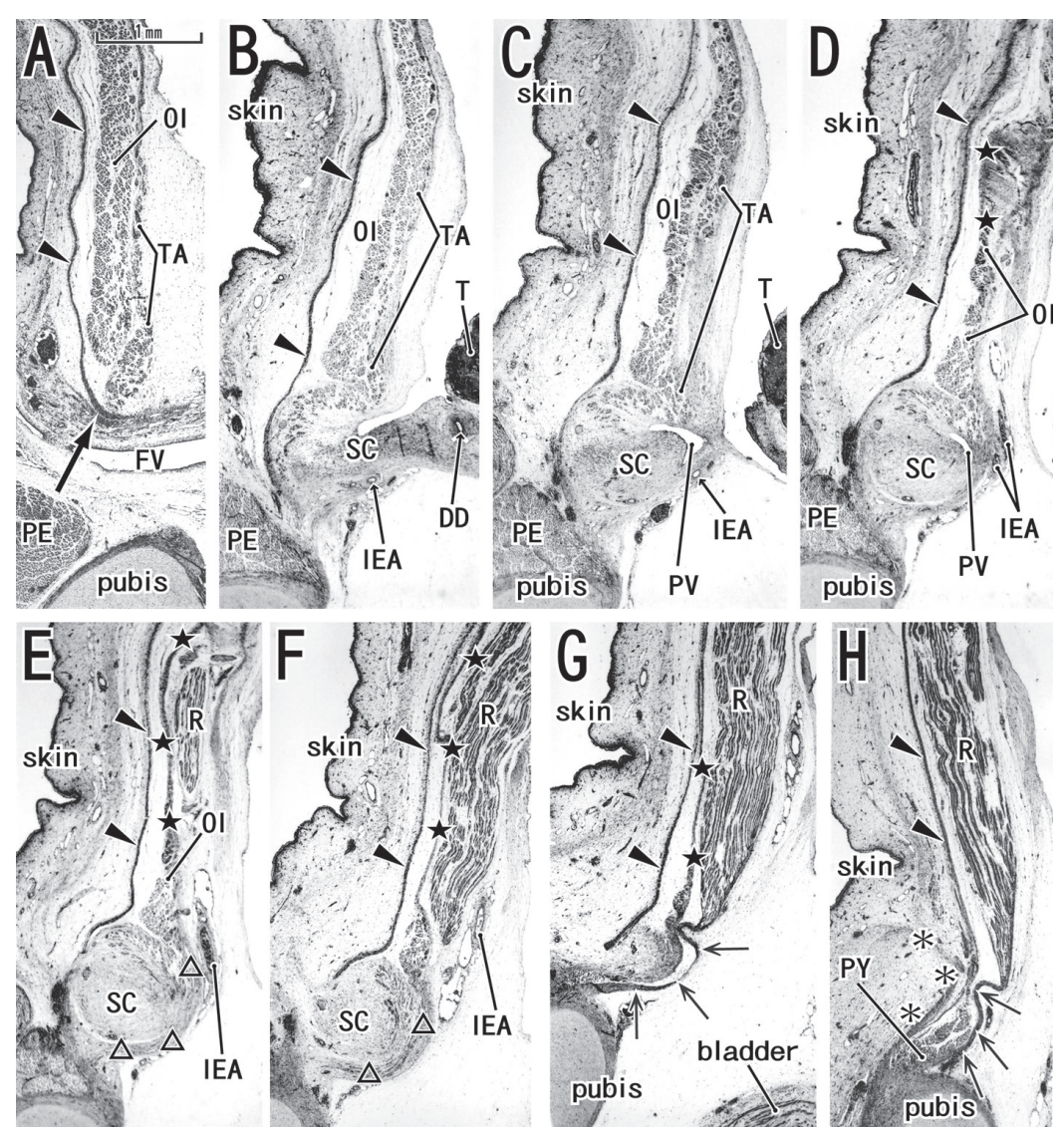

Figure 6. The rectus abdominis tendon extends to the medial side of the superficial inguinal ring in a male foetus. Sagittal sections of a male foetus at approximately 15 weeks of gestation (crown rump length, $115 \mathrm{~mm}$ ) stained with hematoxylin and eosin. Panels $\mathbf{A}$ and $\mathbf{H}$ are the most lateral and medial sites in the figure, respectively. Intervals between panels were $0.8 \mathrm{~mm}(\mathbf{A}-\mathbf{B}), 0.3 \mathrm{~mm}(\mathbf{B}-\mathbf{C}), 0.2 \mathrm{~mm}(\mathbf{C}-\mathbf{D}, \mathbf{D}-\mathbf{E}), 0.3 \mathrm{~mm}(\mathbf{E}-\mathbf{F}), 0.4 \mathrm{~mm}$ $(\mathbf{F}-\mathbf{G})$ and $0.6 \mathrm{~mm}(\mathbf{G}-\mathbf{H})$. In panel $\mathbf{A}$, the most inferior part of the obliquus externus aponeurosis (arrowheads) corresponds to the inguinal ligament (long arrow) covering the femoral vein (FV). Panels $\mathbf{B}$ and $\mathbf{F}$ contain the deep and superficial inguinal rings, respectively. The rectus muscle (R) is not observed on the left-right level of the deep inguinal ring (panel B). The conjoint tendon (stars) extends inferiorly on the deep side of the external obliquus aponeurosis, continuing to a thick fascia (triangles in panels $\mathbf{E}$ and $\mathbf{F}$ ) along the inferoposterior aspect of the gubernaculum or putative spermatic cord (SC). The rectus abdominis tendon (arrows in panels $\mathbf{G}$ and $\mathbf{H}$ ) extends inferiorly on the medial side of the superficial inguinal ring, whereas the external obliquus aponeurosis forms a reflected part (asterisks) on the superficial side of the pyramidalis muscle (PY). All panels were prepared at the same magnification (scale bar in panel A); DD — ductus deferens; T — testis. Other abbreviations are the same as in Figure 3.

insertion of the ligament overlapping a lateral part of the rectus tendon insertion to the pubis. The ligament of Henle was separated from and located in the deep side of the conjoint tendon. Thus, the usual confusion between the ligament and the conjoint tendon (see the Introduction) seemed to be unlikely in foetuses. However, it was too short in the supero-inferior axis to identify easily in sagittal sections. Conversely, as Niikura et al. [11] suggested in relation with a development of the iliopubic tract, a contribution of the covering fascia of the iliopsoas muscle to the posterior wall reinforcement of the inguinal canal may be a male specific morphology. Gilroy et al. [7] reported a slight difference in the iliopubic tract morphology between US and China. Thus, the morphology of the ligament of Henle was also likely to be different between human populations.
Is the suggested gender difference in morphology of the ligament of Henle a result of sexual differentiation in the gubernaculum? However, in the stages examined, the gubernaculum was not so different in size (thickness) between genders. The period of female gonadal descent is much shorter than in males $[3,10]$, i.e., around 33 weeks, although this stage is much later than that of the present specimens. This short period of descent in females might be connected with the presence of the ligament of Henle. The thick gubernaculums is believed to make the large superficial ring in the male inguinal canal [4]. However, to our knowledge, a description on the gender difference in the inguinal canal posterior wall was limited to Rosen et al. [12]. According to them, the women's rectus abdominis muscle was statistically wider than 
men's and, in addition to the other structures such as the conjoint tendon; it can mechanically support the posterior wall of the inguinal canal. Actually, in the present observations, the female rectus consistently extended laterally to the deep inguinal ring: such a foetal topographical anatomy was quite different from adults. Therefore, the mechanical stress from the wider rectus may provide the ligament of Henle at the inferolateral margin in which the developing gubernaculum "attacks" the muscle.

Although Rosser [13] denied its existence, Niikura et al. [11] clearly demonstrated that, in large foetuses at 20-25 weeks, the obliquus internus and transversus abdominis muscles were inserted into a common aponeurosis. We also identified the conjoint tendon in both horizontal and sagittal sections: the tendon was originated from the inferomedial margins of the obliquus internus and transversus abdominis muscles (largely the former). The observations in the present horizontal sections were consistent with Yang et al. [18] who demonstrated that, in human foetuses, the thick insertion aponeurosis of the obliquus internus muscle joins the anterior rectus sheath. However, they failed to show the sagittal section morphology: the conjoint tendon extended inferiorly, became thick and loose, contained striated muscles (parts of the cremaster muscle [11]) and winded around the spermatic cord or the round ligament. The rectus tendon as well as the ligament of Henle supported the posterior aspect of the winding conjoint tendon. Thus, in fetuses, the strong rectus tendon seemed to play a critical role for the posterior wall reinforcement of the inguinal canal.

Sato [14] postulated that, to make clear the origin and lamination of the fibrous tissues of the posterior wall of the inguinal canal, the name "falx inguinalis" is suitable to apply for a structure corresponding to the ligament of Henle. However, in contrast to foetuses, it seems to be difficult to discriminate the adult rectus-associated tissue from the obliquus internus (or tranversus)-associated tissues (e.g. the conjoint tendon) because of postnatal fascial fusion including pathology. Nevertheless, we believe that the difficulty in understanding of the adult morphology does not impair our trial for reappraisal of the ligament of Henle.

\section{ACKNOWLEDGEMENTS}

This study was supported by a grant (0620220-1) from the National R and D Program for Cancer Control, Ministry of Health and Welfare, Republic of Korea.

\section{REFERENCES}

1. Abe $\mathbf{S H}$, Rhee $\mathrm{SK}$, Osonoi $\mathrm{M}$, Nakamura $\mathrm{T}$, Cho $\mathrm{BH}$, Murakami G, Ide $Y$ (2010) Expression of intermediate filaments at muscle insertions of human fetuses. J Anat, 217: 167-173.

2. Anson BJ, Morgan EH, McVay CB (1960) Surgical anatomy of the inguinal region based on a study of 500 body-halves. Surg Gynecol Obstet, 111: 707-725.

3. Backhouse KM (1964) The gubernaculums testis Hunteri: testicular descent and maldescent. Ann R Coll Surg Engl, 35: 15-33.

4. Biasutto SN, Repetto E, Aliendo MA, Borghino VN (2009) Inguinal canal development: the muscular wall and the role of the gubernaculums. Clin Anat, 22: 614-618.

5. Bouchet A, Cuilleret J (1991) Anatomie topographique, descriptive et fonctionnelle, 4 . L'abdomen, la region retro-péritonéale, le petit bassin, le périnée. $2^{\text {nd }} \mathrm{Ed}$. Simep, Paris, pp. 1841-1843.

6. Condon RE (1995) The anatomy of the inguinal region and its relation to groin hernia. In: Nyhus LM, Condon RE eds. Hernia. Chapter 2. $4^{\text {th }}$ Ed. JB Lippincott, Philadelphia, pp. 16-72.

7. Gilroy AM, Marks SC Jr, Lei Q, Page DW (1992) Anatomical characteristics of the iliopubic tract: implications for repair of inguinal hernias. Clin Anat, 5: 255-263.

8. Henle J (1871) Handbuch der systemmatischen Anatomie des Menschen. Bd 1. Teil 3. $2^{\text {nd }}$ Ed. Friedrich Vieweg, Braunschweig, p. 72.

9. Hollinshead H (1966) Anatomy for surgeons. Vol. 2. The thorax, abdomen, and pelvis. Hoeber-Harper International edition. Harper \& Row, New York, pp. 245-246.

10. Heyns CF (1987) The gubernaculums during testicular descent in the human fetus. J Anat, 153: 93-112.

11. Niikura H, Okamot S, Nagase S, Takano T, Murakami G, Tatsumi H, Yaegashi N (2008) Fetal development of the human gubernaculum with special reference to the fasciae and muscles around it. Clin Anat, 21: 547-557.

12. Rosen A, Nathan H, Luciansky E, Orda R (1989) The inguinal region: anatomic differences in men and women with reference to hernia formation. Acta Anat, 134: 306-310.

13. Rosser J (1994) The anatomical basis for laparoscopic hernia repair revisited. Surg Laparoscopy Endoscopy, 4: $36-44$.

14. Sato T (1992) Regional anatomy for operative surgery of genito-urinary organs-34, inguinal region-1. Rinsho-Hinyouki, 46: 198-206.

15. Sen T, Ugurlu C, Kulacoglu $H$, Elhan A (2011) Falx inguinalis: a forgotten structure. ANZ J Surg, 81: 112-113.

16. Skandalakis JE, Colborn GL, Androulakis JA, Skandalakis $\sqcup$, Pemberton LB (1993) Embryology and anatomic basis of inguinal herniorrhaphy. Surg Clin North Am, 4: 799-836.

17. Standring S (2005) Gray's anatomy. $39^{\text {th }}$ Ed. Elsevier Churchill Livingstone, London pp. 1109-1111.

18. Yang JD, Hwang HP, Kim JH, Rodríguez-Vázquez JF, Abe S, Murakami G, Cho BH (2012) Development of the rectus abdominis and its sheath in the human fetus. Yonsei Med J, 53: 1028-1035.

19. Williams PL (1995) Gray's anatomy. $38^{\text {th }}$ Ed. Churchill Livingstone, Edinburgh, p. 825. 